\title{
PRODUCCIÓN CIENTÍFICA ESPAÑOLA EN EL CAMPO DE LOS TRANSPLANTES: 1990-1996
}

\author{
Ellsa Prieto Castro*, Marcos Antonlo Catalán Vega**
}

Resumen: Este estudio bibliométrico intenta dar a conocer la situación que recientemente ha atravesado la investigación española en el campo de los transplantes tanto a nivel nacional como internacional, particularmente en este último. Para determinar esta situación, se han estudiado los documentos publicados por los científicos españoles entre 1990 y 1996 recogidos por las bases de datos IME, SCI y Medline. Además, se ha estudiado el índice de ganancia en competitividad y el índice de actividad de la producción española, comparándolos con la producción internacional y con la de otros parses determinados. Por otro lado, se ha realizado un estudio de la bibliografía utilizada por los cientificos españoles, mediante diversos indicadores como la vida media de las publicaciones y el índice de Price de referencias y citas. Los resultados obtenidos indican que la producción española está en un momento de auge, aunque tiene una tendencia excesivamente irregular, y aunque se han observado muchas diferencias respecto a los países más importantes, los puntos a favor encontrados matizan esta opinión desfavorable.

Palabras clave: bibliometría, transplantes, vida media, índice de Price, ganancia en competitividad, índice de actividad, ventana de citación, redes de colaboración.

Abstract: The aim of this bibliometric study is to find out the state of Spanish research in the field of transplantation at the national and, specially, international level. Documents published from 1990-1996 by Spanish researchers were collected from IME, SCI and Medline databases. Two different indexes were calculated: the Gain in Competitiveness and the Activity Index, which compare both the Spanish research versus world production, and versus high productive countries. Information use was studied using the analysis of references from the articles by Spanish researchers, their Half-Life and Price's Index. The results show that research in the Transplantation field is in a period of expansion worldwide. Big differences exist between Spanish production and the production of those countries that may be considered as big producers, but the overall conclusion is positive.

Keywords: bibliometrics, transplantation, activity index, half-life, Price's index, gain in competitiveness, citation window, collaboration nets.

* Elzaburu S.A. Correo-e: elisa_prieto@hotmail.com

** Universidad Carlos III de Madrid. Correo-e: marcoscv@ hotmail.com

Recibido: 20-1-99. Segunda versión: 14-10-99. 


\section{Introducción}

España es uno de los países punteros en donación y transplante de órganos. Las cifras y porcentajes que nos legan desde la Organización Nacional de Transplantes (1) así lo confirman. Al mismo tiempo, los medios de comunicacion se hacen eco del gran número de donaciones que se realizan en nuestro país y de la alta tasa de éxito de las operaciones; factor que, a su vez, resulta estimulante de otras muchas nuevas (2).

De aquí surge el interés en determinar si este éxito de la medicina española tiene un reflejo semejante en el volumen de producción científica de nuestros profesionales.

Por ello, la investigación se circunscribe a los trabajos realizados por investigadores españoles, aunque también se han recogido los datos de algunos otros países para contextualizar esta producción en el ámbito internacional.

El período de estudio comprende los años que van desde 1990 hasta 1996 . Se estima que es un ciclo suficientemente dilatado para establecer la evolución de los resultados y lo bastante actualizado para resultar de interés.

Los objetivos iniciales del trabajo son analizar la producción y evolución de la investigación española dentro del ámbito de los transplantes, tanto en el ámbito nacional como internacional. También se pretende determinar el grado de colaboración científica existente y la actualización de la literatura utilizada por los autores españoles, viendo si estos datos cambian en función del ámbito de publicación del artículo.

\section{Metodología}

Se realizó una serie de búsquedas correspondientes a los documentos relativos al período de 1990 a 1996 en las bases de datos Medline, Índice Médico Español (IME) y Science Citation Index (SCI). Para Medline se usó el CD-ROM distribuido por Silverplatter, en la versión actualizada en diciembre de 1997. Para el IME el CD-ROM distribuido por el CSIC con fecha de abril de 1998 y para el SCI los CD-ROM anuales.

Los datos recogidos en las bases de datos Medline y $\mathrm{SCI}$ fueron el número total de documentos de la base de datos, el número total de documentos sobre transplantes, el número total de documentos de cada uno de los países seleccionados y el número total de documentos sobre transplantes por país. Asimismo, el dato recogido de la base de datos IME fue el número total de documentos sobre transplantes.

En la realización de la búsqueda documental, se contó con el apoyo de un bibliotecario especializado en documentación médica. Así, la búsqueda en la base de datos IME se realizó a través del campo «descriptores» utilizando vocabulario específico, extraído y traducido del $\mathrm{MeSH}$ y de diversas obras de referencia de carácter especializado. A modo de ejemplo, del Diccionario Médico Roche (editorial Doyma) se extranjeron conceptos como «transplante singenético», «transplante alogenético», «transplante xenogenético», "pulmonar», etc. De igual modo, en Medline, la búsqueda por tema se realizó a través del $\mathrm{MeSH}$. Para ello, en el campo del mismo nombre, se tuvieron en cuenta todos los tipos de transplantes, y un gran número de descriptores relacionados que este tesauro contempla. Por otro lado, en la búsqueda dentro del SCI se introdujeron todos los conceptos extraídos del $\mathrm{MeSH}$ en el campo título, ya que esta base de datos no dispone de un campo relativo a temática, sea controlada o no.

La base de datos SCI, a pesar de ser mucho más genérica que Medline, arrojó unos resultados muy parecidos a esta última. Así, dentro de esta temática, el SCI solapa a Medline en un $43,33 \%$, siendo éste un valor relativamente alto. También se ha obser- 
vado que los documentos encontrados en esta base pueden considerarse un buen complemento a los de Medline, pues hay una tipología documental algo distinta. De igual modo, IME solapa a Medline en un $53,76 \%$, lo que puede indicar que Medline, a pesar de su carácter internacional, dispone de una buena cobertura de la producción publicada en España, recogida en el Indice Médico Español. Por contra, el solapamiento que se produce entre las bases de datos SCI e IME es muy bajo.

Se puso especial cuidado en la búsqueda por países, especialmente en lo referente a España y Estados Unidos, para así conseguir una recuperación lo más exhaustiva y precisa posible. Hay que tener en cuenta que dos de las bases de datos son producidas en EE.UU. y que recogen especialmente artículos de revistas y autores pertenecientes al ámbito anglosajón. Esto hace que a menudo no conste de forma explícita el nombre del país norteamericano, sino sólo el del estado o ciudad, a veces incluso en forma de siglas que, en ocasiones, pueden generar un alto volumen de ruido (por ejemplo: DE - Delaware, IN - Indiana o LA - Los Angeles). En el caso de España, se incluyeron todos los nombres de provincias y ciudades importantes, siempre poniendo atención a los homónimos (Córdoba - Argentina, Santiago - Chile, Guadalajara - México, Valencia - Venezuela o Laguna - Filipinas).

Los datos sobre recursos económicos dedicados a investigación médica han sido extraídos de las memorias del Fondo de Investigación Sanitaria, en el apartado de inversiones en proyectos de investigación (3). Se han recopilado los datos relativos al período 1988 - 1995 pues se entiende que las inversiones empiezan a tener resultados bibliográficos cierto tiempo después de ser concedidas.

De igual modo, para determinar si una revista tiene un ámbito de difusión nacional o internacional, se han tenido en cuenta, entre otros, algunos factores como son el idioma de publicación, la intencionalidad expresada por la propia publicación y la opinión de un bibliotecario experto en documentación médica.

Por último, es preciso indicar que se han utilizado dos formulas para el estudio de los resultados: el indicador de ganancia en competitividad propuesto por Cano y Julián (4) para cuantificar la evolución de la producción española y el índice de actividad planteado por Frame (5) y utilizado por Guzmán, Sanz y Sotolongo (6), Schubert y Braun (7), y Nagpaul y Sharma (8) como herramienta en la comparación de la producción de los diferentes países. El primero de ellos se desarrolla con la expresión $\left[(\mathrm{PE} / \mathrm{PR})_{e} /(\mathrm{PE} / \mathrm{PR})_{b}\right]-1$, expresado en porcentaje; donde PE y PR indican, respectivamente, la producción científica de España y la del resto del conjunto de referencia en el año evaluado (e) y en el año base (b). El segundo índice presenta la expresión $\left[\left(\mathrm{N}_{\mathrm{ij}} / \mathrm{N}_{\mathrm{i} 0}\right) /\left(\mathrm{N}_{0 \mathrm{j}} / \mathrm{N}_{00}\right)\right]$; donde $\mathrm{N}_{\mathrm{ij}}$ indica el número de documentos publicados por un país sobre una disciplina en concreto, $\mathrm{N}_{\mathrm{i} 0}$ es el número de documentos publicados por un país, $\mathrm{N}_{0 \mathrm{j}}$ señala el número de documentos publicados por todos los países sobre una disciplina en concreto y $\mathrm{N}_{00}$ es el número total de documentos publicados por todos los países en todas las disciplinas.

\section{Resultados y discusión}

\subsection{Producción científica de los autores españoles}

En la tabla I constan los valores totales de los documentos recuperados en las ba- 
ses de datos IME, Medline y SCI. Los datos corresponden al total de documentos españoles sobre transplantes.

Tabla I

Produccion total. Datos de IME, Medline y SCI

\begin{tabular}{|c|c|c|c|}
\hline Afio & $\begin{array}{c}\text { Documentos } \\
\text { españoles sobre } \\
\text { transplantes en } \\
\text { IME }\end{array}$ & $\begin{array}{c}\text { Documentos } \\
\text { españoles sobre } \\
\text { transplantes en } \\
\text { Medline }\end{array}$ & $\begin{array}{c}\text { Documentos } \\
\text { españoles sobre } \\
\text { transplantes en } \\
\text { SCI }\end{array}$ \\
\hline 1990 & 100 & 125 & 85 \\
1991 & 71 & 118 & 90 \\
1992 & 85 & 200 & 146 \\
1993 & 103 & 177 & 163 \\
1994 & 158 & 216 & 146 \\
1995 & 221 & 297 & 225 \\
1996 & 200 & 248 & 214 \\
\hline Total & 938 & 1.381 & 1.069 \\
\hline
\end{tabular}

Se puede apreciar cómo la producción española ha ido aumentando año tras año, aunque de forma diferente y significativa según la base de datos. Por ejemplo, dentro de la base de datos Medline, los documentos sobre transplantes originarios de nuestro país llegan a subir un $\mathbf{9 8 , 4 \%}$, mientras que en el IME aumentan un $100 \%$ y en el SCI crecen el $151,76 \%$. Esta cifra es aún más significativa si la comparamos, por ejemplo, con el $31,02 \%$ en que se ha visto aumentada la representación total de documentos sobre transplantes en el total de la base de datos Medline (de 7.104 documentos en 1990 se pasa a 9.308 en 1996).

Asimismo, los valores de las tres bases de datos son fluctuantes; aunque el pico de resultados se da siempre en 1995, produciéndose un descenso en 1996.

En la tabla II se representa la ganancia en competitividad realizada según la fórmula indicada anteriormente. Para ello, se han utilizado los valores de la base de datos Medline por su carácter internacional, de cara a compararlos con la producción de otros países dentro del mismo medio (completar estos datos con registros del IME o del SCI sesgaría el análisis). Así, gracias a estos cálculos se puede cuantificar la evolución de la producción española con relación a todo lo recopilado por esta base de datos internacional. Mientras que la producción española total muestra una subida leve pero continuada año tras año, no ocurre lo propio con el conjunto de la base de datos en el campo de los transplantes. La aportación en este campo presenta continuos altibajos, con subidas muy acusadas e incluso valores negativos respecto al año base (por ejemplo: $90,79 \%$ en 1995 y $-8,71 \%$ en 1991). La tendencia, en cualquier caso, es al alza y con valores más elevados respecto al total de los artículos españoles.

Además, los datos extraídos de las diferentes bases de datos muestran que el científico español tiende, cada vez más, a publicar en el extranjero. Este fenómeno repercutirá, en un futuro próximo, en que la investigación española en el campo de los transplantes sea cada vez más conocida y tenga una mayor difusión, ya que las publicaciones españolas parecen no gozar de toda la relevancia internacional que sería deseable. 
Tabla II

Ganancia en competitividad de la producción española. Datos de Medline

\begin{tabular}{|c|c|c|c|c|c|c|}
\hline Año & $\begin{array}{c}\text { Documentos } \\
\text { españoles sobre } \\
\text { transplantes }\end{array}$ & $\begin{array}{c}\text { Resto de } \\
\text { documentos de } \\
\text { transplantes }\end{array}$ & G.C. & $\begin{array}{c}\text { Total de } \\
\text { documentos } \\
\text { españoles }\end{array}$ & $\begin{array}{c}\text { Resto de } \\
\text { documentos en } \\
\text { Medline }\end{array}$ & G.C. \\
\hline 1990 & 125 & 6.979 & - & 5.672 & 371.021 & - \\
1991 & 118 & 7.216 & -8.71 & 5.850 & 369.962 & 3.43 \\
1992 & 200 & 7.455 & 49,78 & 6.463 & 370.842 & 14 \\
1993 & 177 & 8.028 & 23,09 & 6.697 & 375.701 & 16.6 \\
1994 & 216 & 8.727 & 38,18 & 6.771 & 381.564 & 16.07 \\
1995 & 297 & 8.691 & 90,79 & 7.553 & 386.766 & 27,74 \\
1996 & 248 & 9.060 & 52.82 & 8.306 & 385.721 & 40,85 \\
\hline
\end{tabular}

\subsection{Producción comparada con otros países}

Al comparar, por medio de porcentajes totales, la producción científica de España con otros países de nuestro entorno, Japón y EE.UU., se ve que la contribución española no es desdeñable (véase figura 1) y que va en aumento, sobre todo si la comparamos, por ejemplo, con el $0,92 \%$ que exponían $\mathrm{M}$. A. Pérez San-Gregorio et al. en un estudio relativo a la producción sobre aspectos psicológicos implicados en las donaciones que abarca el período comprendido entre 1972 y 1993 (9).

Figura 1

Producción mundial de publicaciones $(1990$ - 1996) sobre transplantes

Datos de MEDLINE

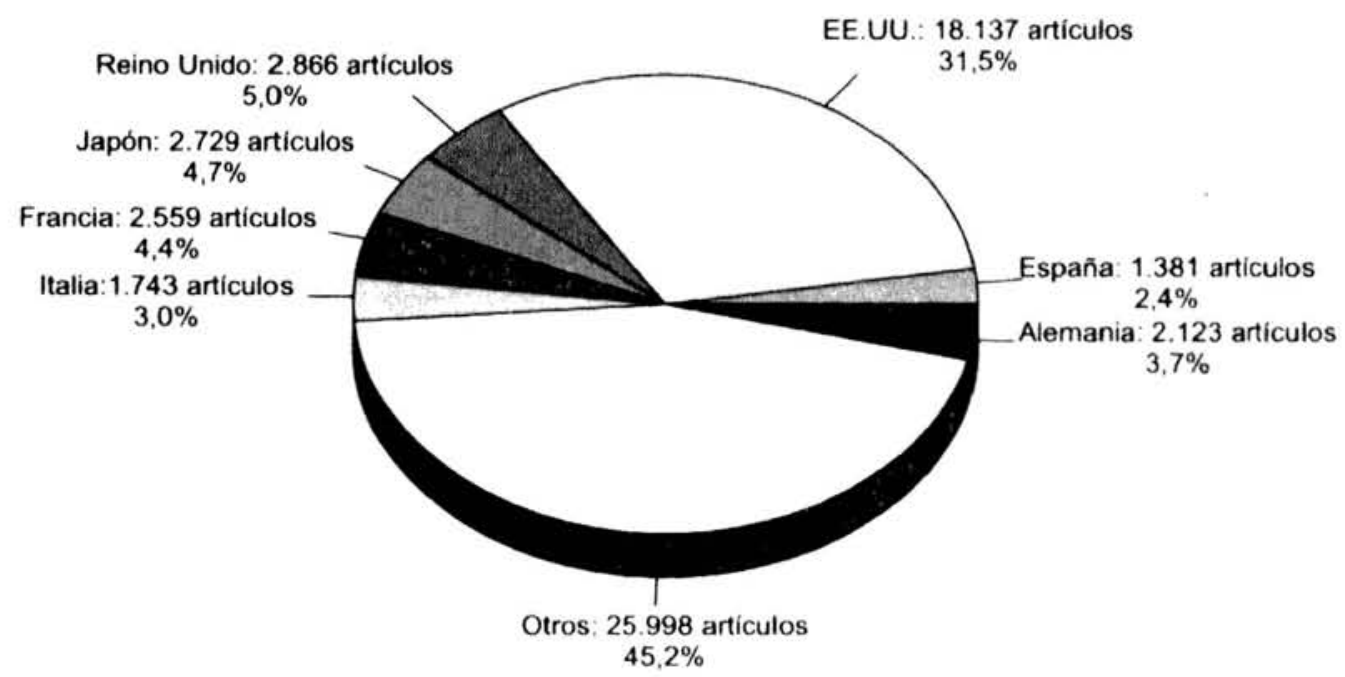

De igual forma, el estudio de la ganancia en competitividad muestra algunos datos significativos (véase tabla III).

En primer lugar, los países fuera del marco europeo muestran tendencias muy diferentes. El caso americano es propio de los países en una fase científica madura con producción elevada, tal y como comentan, entre otros, Goldberg, Oigenblick y Rubin 
(10). Japón, sin embargo, se encuentra dentro de los países que aumentan drásticamente su aportación, pasando de producir menos que algunos países europeos a situarse prácticamente a la cabeza de todos ellos. Su ganancia en competitividad sigue una línea ascendente continua que sólo se puede comparar a la alemana. El caso japonés podría encuadrarse dentro de los países en fase muy activa de desarrollo científico que inician su andadura con una representación modesta en el sector y tienen aumentos muy significativos a lo largo de los años.

\section{Tabla III}

Comparación de la ganancia en competitividad en artículos sobre transplantes Datos de MEDLINE

\begin{tabular}{|c|c|c|c|c|c|c|c|c|}
\hline$A \tilde{n} o$ & & España & EE.UU. & Reino Uni. & Japón & Francia & Italia & Alemania \\
\hline \multirow[t]{2}{*}{1990} & $\begin{array}{l}\text { Artículos } \\
\text { del pais }\end{array}$ & 125 & 2.047 & 332 & 235 & 268 & 178 & 124 \\
\hline & G.C. & - & - & - & - & - & - & - \\
\hline \multirow[t]{2}{*}{1991} & $\begin{array}{l}\text { Artículos } \\
\text { del país }\end{array}$ & 118 & 2.164 & 392 & 285 & 266 & 266 & 195 \\
\hline & G.C. & $-8,7$ & 3,4 & 15,18 & 18,17 & -4 & 46,43 & 53,75 \\
\hline \multirow[t]{2}{*}{1992} & $\begin{array}{l}\text { Artículos } \\
\text { del país }\end{array}$ & 200 & 2.177 & 335 & 329 & 291 & 185 & 248 \\
\hline & G.C. & 49,78 & $-1,82$ & $-6,65$ & 31,26 & 0,79 & $-3,63$ & 88,47 \\
\hline \multirow[t]{2}{*}{1993} & $\begin{array}{l}\text { Artículos } \\
\text { del pais }\end{array}$ & 177 & 2.663 & 418 & 386 & 387 & 278 & 330 \\
\hline & G.C. & 23,09 & 18,7 & 9,49 & 44,29 & 26,26 & 36,45 & 135,88 \\
\hline \multirow[t]{2}{*}{1994} & $\begin{array}{l}\text { Artículos } \\
\text { del pars }\end{array}$ & 216 & 2.784 & 457 & 473 & 423 & 263 & 384 \\
\hline & G.C. & 38,18 & 11,68 & 9,86 & 63,25 & 26,65 & 17,9 & 152,57 \\
\hline \multirow[t]{2}{*}{1995} & $\begin{array}{l}\text { Artículos } \\
\text { del país }\end{array}$ & 297 & 3.145 & 453 & 443 & 503 & 257 & 377 \\
\hline & G.C. & 90,79 & 32,97 & 8,26 & 51,53 & 51,21 & 14,53 & 146,44 \\
\hline \multirow[t]{2}{*}{1996} & $\begin{array}{l}\text { Artículos } \\
\text { del país }\end{array}$ & 248 & 3.157 & 479 & 578 & 421 & 316 & 465 \\
\hline & G.C. & 52,82 & 26,79 & 10,66 & 93,52 & 20,83 & 36,73 & 195,99 \\
\hline
\end{tabular}

Dentro de los países europeos, el caso alemán es especialmente señalado. Existe un aumento continuado de ganancia en competitividad respecto al año base que llega a porcentajes muy altos $(195,99 \%$ en el año 1996). Esto es más relevante aún si se considera que su producción era la más modesta, por debajo también de España, en 1990. La reunificación de las dos Alemanias puede ser la base de este cambio. Aunque en la búsqueda se tuvo cuidado de incluir las dos denominaciones del país (República Federal y República Democrática) para los primeros años, cuando aún eran dos estados, es lógico pensar que los científicos orientales publicaran mucho menos en revistas de occidente que en las del antiguo bloque comunista, que a su vez están menos representadas en Medline. El contar, hoy en día, con una política común de investigación puede también haber ayudado mucho. 
El caso británico podría considerarse el homólogo europeo de EE.UU. La producción en este país es, desde su inicio, la más elevada en el continente y termina también con la cifra más alta (aunque seguida de cerca por Alemania). Sin embargo, su aumento en competitividad es algo bajo; lo que hace pensar que quizás haya llegado a un punto de saturación.

Por último, Francia, Italia y España presentan una situación bastante similar en cuanto a su producción y aumento de competitividad. Todos ellos cuentan con una trayectoria irregular, aunque en el caso francés es menos pronunciada que en España o Italia. España es el país con mayor ganancia en competitividad de los tres, pero también el de tendencia más inestable. Este dato puede corroborar una cierta inmadurez investigadora propia de países con financiación fluctuante, que varía según las prioridades de la política de investigación (11).

Como conclusión a este análisis se puede recurrir a la línea de tendencia de los artículos publicados para compararla con la aportación de cada uno de los países (véase figura 2). De este modo, se observan más claras las corrientes que ya se han podido vislumbrar en la prueba de la ganancia en competitividad.

Figura 2

Tendencia de documentos publicados sobre transplantes en los países europeos Datos de MEDLINE

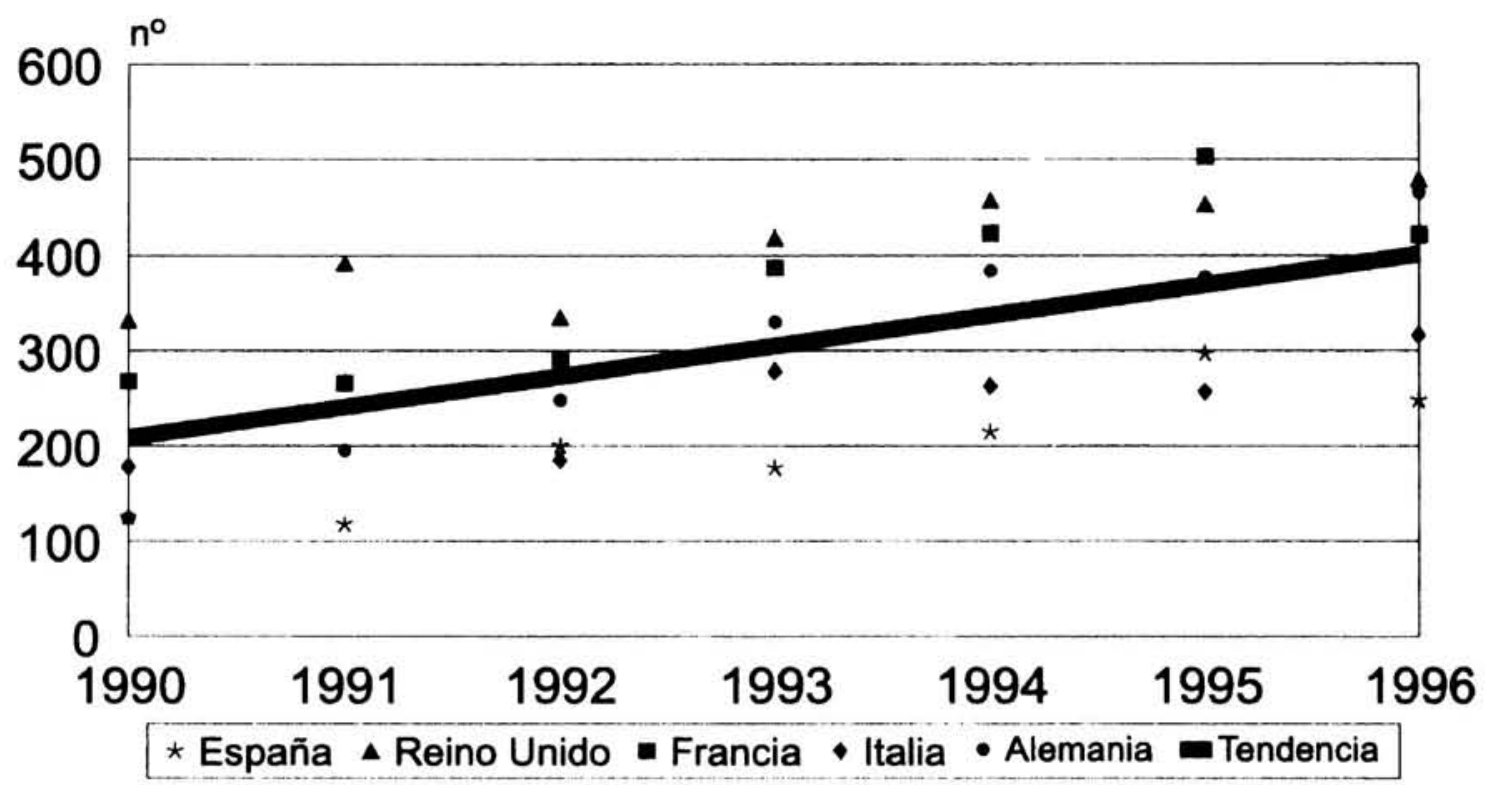

Italia y España son los países que se encuentran por debajo de la media en todo el espectro temporal. Ambos muestran una fluctuación importante en los resultados, siendo Italia la que ofrece, casi siempre, mejores rendimientos. Es curioso observar cómo las subidas y bajadas de ambos países no coinciden nunca, ni entre sí ni con la línea general de promedios. Esto hace descartar cualquier acontecimiento que haya influido en la producción de todos los países, por ejemplo, una bajada de los artículos indizados.

Por encima de la media se sitúan siempre Francia y el Reino Unido, con unas ten- 
dencias mucho más equilibradas que las anteriores. Aunque también presentan fluctuaciones, sus líneas tienen una dirección ascendente.

El caso más espectacular es el alemán, que ofrece una línea casi totalmente ascendente, desde unos inicios muy modestos hasta terminar prácticamente en cabeza de todos los países mostrados.

Si a estos análisis se les afiaden los resultados obtenidos relativos al índice de actividad (véase tabla IV), se observa que, efectivamente, el tema de los transplantes tiene una alta prioridad investigadora en España si comparamos ésta con el resto de los países (tan sólo en Francia se le otorga a la materia una importancia mayor).

\section{Tabla IV}

\section{Indice de actividad. Datos de SCI y MEDLINE}

\begin{tabular}{|l|c|}
\hline \multicolumn{1}{|c|}{ Pats } & Indice de actividad \\
\hline Espafia & $138,09 \%$ \\
EE.UU. & $109,52 \%$ \\
Reino Unido & $104,76 \%$ \\
Japón & $76,19 \%$ \\
Francia & $152,38 \%$ \\
Italia & $123,8 \%$ \\
Alemania & $133,33 \%$ \\
Resto de los parses & $90,47 \%$ \\
\hline
\end{tabular}

\subsection{Distribución geográfica. Centros productores y comparación autonómica}

Este estudio se ha realizado a partir de los resultados obtenidos sólo de la base de datos Medline, pues se trata de diferenciar aquellas comunidades autónomas con científicos que publican en revistas de ámbito internacional, intentando distinguir los hospitales y comunidades con mayor presencia fuera de las fronteras de nuestro país. (Los documentos se han asignado al centro del primer autor porque Medline tan sólo incluye su dirección.)

Así, como se aprecia en la tabla V, los resultados desglosados por comunidades autónomas no resultan demasiado sorprendentes en lo relativo a los primeros puestos: $\mathrm{Ca}$ taluña y Madrid. No es extraño que tales zonas, que cuentan con un número muy elevado de centros hospitalarios y que reciben aportaciones monetarias cuantiosas para investigación médica, ofrezcan una producción bibliográfica elevada. No se debe tampoco perder de vista que sólo ciertos hospitales en el país, que poseen el equipo humano y técnico necesario así como los permisos legales, pueden realizar transplantes.

Este hecho puede estar en la base de la escasa representación de ciertas comunidades que no cuentan con tales instalaciones y que, por lo tanto, deben acudir a hospitales en otras ciudades. Este es un dato a tener muy presente cuando se descubren ciertos centros de excelencia que acaparan, en ocasiones, toda la producción regional u ofrecen unas cifras destacadas (3).

El análisis de los siguientes puestos muestra algunas sorpresas, no sólo por la gran diferencia respecto a los dos primeros, sino también porque variables como el número de habitantes no parecen afectar a los resultados, ya que regiones como Navarra 
Tabla V

Producción total por CC.AA. Datos de MEDLINE

\begin{tabular}{|l|r|r|r|r|r|r|r|r|}
\hline \multicolumn{1}{|c|}{ CC.AA. } & 1990 & 1991 & 1992 & 1993 & 1994 & 1995 & 1996 & Total \\
\hline 4. Andalucía & 6 & 4 & 15 & 14 & 17 & 15 & 15 & 86 \\
13. Aragón & 0 & 0 & 2 & 0 & 3 & 1 & 3 & 9 \\
12. Asturias & 2 & 2 & 2 & 0 & 3 & 1 & 1 & 11 \\
14. Baleares & 0 & 0 & 0 & 0 & 1 & 2 & 3 & 6 \\
11. Canarias & 1 & 0 & 4 & 2 & 2 & 3 & 3 & 15 \\
6. Cantabria & 5 & 10 & 10 & 14 & 11 & 8 & 7 & 65 \\
14. Castilla - La Mancha & 0 & 0 & 1 & 0 & 2 & 3 & 0 & 6 \\
10. Castilla - León & 1 & 1 & 3 & 0 & 4 & 6 & 2 & 17 \\
1. Cataluña & 40 & 43 & 71 & 70 & 53 & 104 & 75 & 456 \\
16. Extremadura & 0 & 0 & 1 & 0 & 0 & 1 & 1 & 3 \\
8. Galicia & 0 & 1 & 4 & 4 & 7 & 6 & 9 & 31 \\
2. Madrid & 47 & 36 & 55 & 48 & 59 & 89 & 92 & 426 \\
7. Murcia & 0 & 0 & 2 & 6 & 8 & 13 & 9 & 38 \\
3. Navarra & 10 & 13 & 11 & 9 & 26 & 16 & 8 & 93 \\
9. País Vasco & 3 & 3 & 4 & 1 & 4 & 5 & 3 & 23 \\
17. La Rioja & 0 & 1 & 0 & 0 & 1 & 0 & 0 & 2 \\
5. Valencia & 7 & 3 & 15 & 7 & 12 & 21 & 17 & 82 \\
Ilocalizables & 3 & 1 & 0 & 2 & 3 & 3 & 0 & 12 \\
\hline Total & 125 & 118 & 200 & 177 & 216 & 297 & 248 & 1.381 \\
\hline
\end{tabular}

o Cantabria poseen totales bastante mejores que comunidades como el País Vasco o Aragón. La explicación, como se ha comentado, podría estar en la existencia de centros hospitalarios de excelencia en estas comunidades (véanse tabla VI y figura 3).

En algunas comunidades pequeñas existen centros con una producción muy alta dentro del total de instituciones del país (por ejemplo: Navarra o Cantabria). Por lo general, estos centros constituyen un porcentaje muy alto del total regional y pueden, en al-

Tabla VI

Centros españoles que más documentos publican sobre transplantes. Datos de MEDLINE

\begin{tabular}{|l|c|}
\hline \multicolumn{1}{|c|}{ Centro } & $\begin{array}{c}\text { Número de } \\
\text { documentos }\end{array}$ \\
\hline Hospital Clínic i Provincial Barcelona (Cataluña) & 179 \\
Hospital Universitario 12 de Octubre (Madrid) & 107 \\
Ciutat Sanitaria i Universitaria de Bellvitge (Cataluña) & 90 \\
Hospital Ramón y Cajal de Madrid (Madrid) & 68 \\
Hospital Universitario Marqués de Valdecilla (Cantabria) & 61 \\
Clínica Universitaria (Navarra) & 57 \\
Hospital Universitario La Fe (Valencia) & 56 \\
Clínica Puerta de Hierro (Madrid)* & 51 \\
Hospital General Universitari de la Vall d'Hebrón (Cataluña) & 49 \\
\hline Total del núcleo & 718 \\
\hline
\end{tabular}

* Este centro es el de mayor producción según los baremos de J. A. Cortés Guardiola (12); la diferencia de posición entre su estudio y el presente puede deberse al uso de diferentes bases de datos para extraer los documentos a estudiar. 


\section{Figura 3}

Mapa de comunidades autónomas y principales ciudades. Datos de MEDLINE

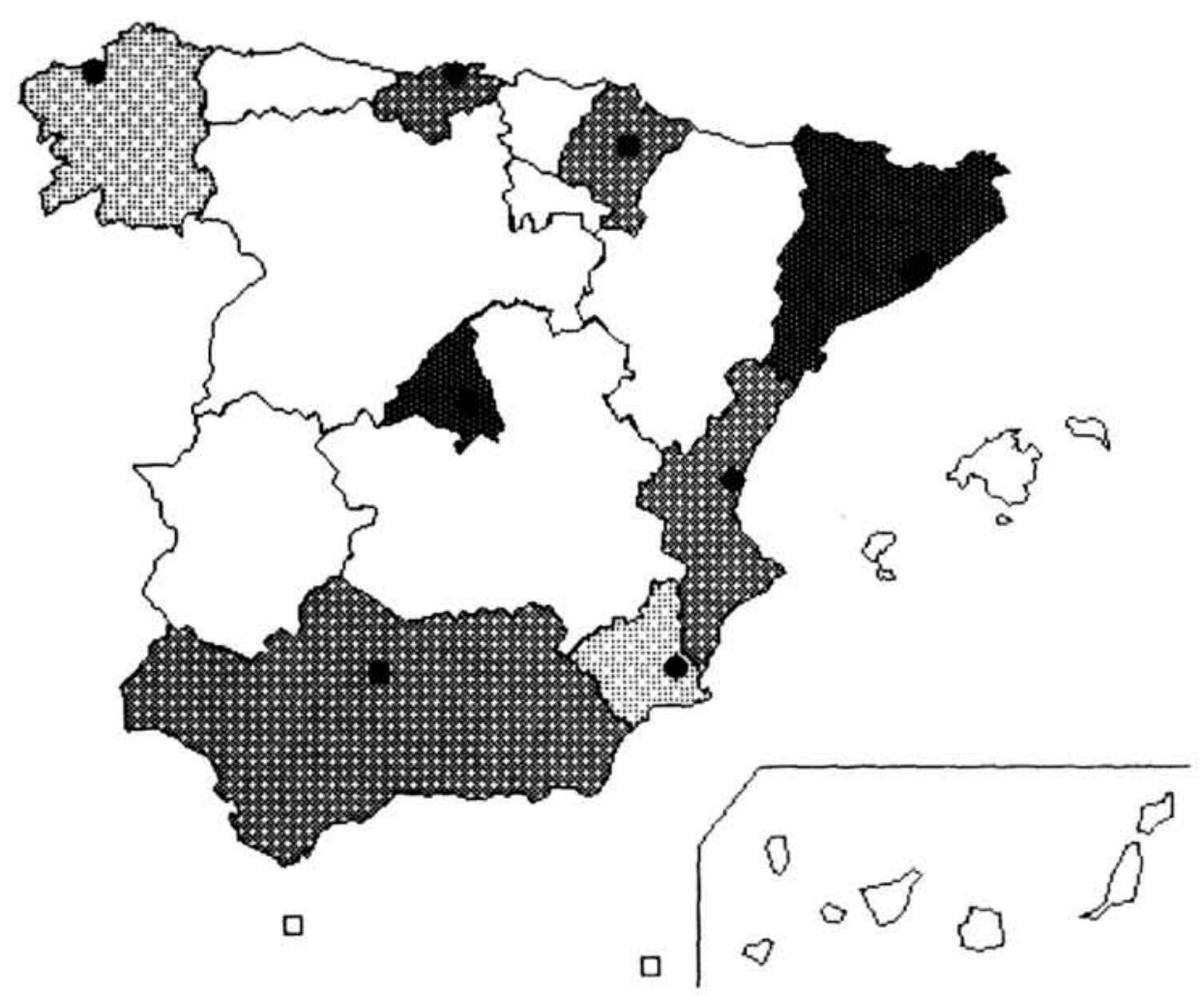

25 artículos o menos

$\square$ de 26 a 50 artículos

圈 de 51 a 100 artículos

más de 100 artículos

- cudades con organizaciones que han publicado 25 artículos o más

gunos casos, suponer casi el $100 \%$ de la zona (por ejemplo: el Hospital Universitario Marqués de Valdecilla sobre el total de la producción en Cantabria). Así, en zonas de territorio tan reducido, es probable que estos centros sean los únicos con la infraestructura y el equipo necesarios para realizar estas intervenciones.

En otras regiones, de territorio no tan reducido, se observa este mismo hecho: un único centro aporta la mayoría de lo publicado. En Galicia, el Complejo Hospitalario Juan Canalejo publica 25 de los 31 artículos con dirección en la comunidad, en Murcia el Hospital Universitario Virgen de la Arraixaca publica 32 de 38 y en Andalucía el Complejo Hospitalario Reina Sofía de Córdoba publica 46 de los 85 artículos.

Por otro lado, conviene apuntar que en algunas comunidades autónomas (en concreto: Madrid, Andalucía y Galicia) el volumen de producción podría estar algo por debajo de lo que cabría esperar del volumen total de fondos destinados a investigación médica; sobre todo si se comparan con otras como son Cataluña, Cantabria, Navarra, Murcia 
y Valencia. (Esto puede deberse a numerosos factores. El más importante de ellos podría ser la diferencia en cuestiones de prioridad del tema en según que comunidades. Esto es así porque, si se tienen en cuenta la totalidad de fondos destinados a la investigación médica de una comunidad respecto a otra, ha de considerarse, concretamente, dónde se destinan estos fondos; ya que una comunidad autónoma que reciba mayores fondos, en conjunto, puede dedicar menos que otra a la investigación en transplantes.)

\subsection{Tipología documental}

Los datos recogidos en todas las bases de datos corroboran que el vehículo de comunicación de los científicos españoles es el artículo científico (por ejemplo, el $75 \%$ del total en Medline y el $84 \%$ en el IME), seguido del artículo de revisión (12,89\% en Medline) y del ensayo clínico ( $11,81 \%$ también en Medline).

En definitiva, queda reflejado que, en esta temática como en la mayoría de las disciplinas experimentales y aplicadas (13), el artículo sigue siendo el vehículo preferido por los investigadores para difundir sus conocimientos. Sin embargo, existen diferencias de vaciado de las bases de datos utilizadas en el estudio. Por ejemplo, tanto en Medline como en el IME y el SCI, el artículo científico es el vehículo más utilizado, aunque con unos resultados muy dispares (84\% en IME, $75 \%$ en Medline y el $53,6 \%$ en SCI). Las mayores desigualdades se encuentran, sin embargo, en el porcentaje de artículos de revisiones y en las comunicaciones a congresos. Mientras que, por ejemplo, Medline llega a un $12,89 \%$ de artículos de revisión, el SCI sólo alcanza el $0,74 \%$; por otro lado, la primera incluye menos comunicaciones a congresos que la segunda (un $0,3 \%$ frente a un $27,22 \%$ ). A ello se une una tipología documental más variada en el caso de SCI: incluye cartas $(10,75 \%)$, notas $(6,36 \%)$, editoriales $(1,05 \%)$ y comentarios $(0,28 \%)$. Por su parte, el IME también recoge un amplio espectro de tipología documental: $7 \%$ de editoriales (siendo el índice más alto de todas las bases de datos), $3 \%$ de ensayos clínicos, $2 \%$ de cartas al director, y un $4 \%$ de documentos repartidos entre otros tipos de comunicación científica.

\subsection{Colaboración entre científicos y centros de investigación}

La colaboración entre científicos se ha calculado mediante el índice de coautoría (véase tabla VII). (Medline incluye sólo los diez primeros autores y en caso que haya alguno más, los indica con la partícula «et al.». Así, se ha decidido que, dentro de los artículos extraídos de esta base de datos, el artículo con más de diez autores se ha registrado como once.)

Los dos valores de coautoría pueden considerarse altos (la media es de 6,02) si se comparan con otras disciplinas médicas como, a modo de ejemplo, el caso del cáncer, que ha mostrado en algunos estudios bibliométricos un índice de coautoría próximo a tres autores (14). Así se constata que es un hecho bastante común, dentro del área de las ciencias aplicadas y experimentales, alcanzar un nivel elevado de cooperación entre autores a la hora de redactar trabajos (12), quizás debido a la necesidad de realizar muchos experimentos en situaciones diferentes. Este hecho cobra especial trascendencia en el campo médico, pues cualquier afirmación debe venir avalada por numerosas pruebas y estudios en pacientes con síntomas diversos. Además, según M.A. Pérez San 
Tabla VII

fndice de coautoría de los trabajos Datos de IME y MEDLINE

\begin{tabular}{|c|c|c|}
\hline Afto & $\begin{array}{c}\text { Indice de coautoria } \\
\text { en imbito nacional }\end{array}$ & $\begin{array}{c}\text { Indice de coautoria en } \\
\text { ambito internacional }\end{array}$ \\
\hline 1990 & 5 & 6,24 \\
1991 & 5,46 & 6,07 \\
1992 & 5,84 & 6,95 \\
1993 & 5,54 & 6,74 \\
1994 & 5,87 & 6,53 \\
1995 & 5,6 & 6,56 \\
1996 & 5,39 & 6,61 \\
\hline Media & 5,52 & 6,52 \\
\hline
\end{tabular}

Gregorio et al., este alto índice puede influir en los valores de producción, ya que se apunta «que los autores más productivos cuentan en su mayoría con un alto índice de colaboración” (15).

De igual modo, resulta interesante comparar los valores de la coautoría en los dos ámbitos estudiados. El hecho de que una revista nacional tenga un índice sensiblemente inferior (de 1 autor por trabajo) puede deberse a que, a la hora de remitir los estudios a las revistas internacionales, se incluya el nombre de todos los miembros de un grupo de investigación o trabajo, no sólo de los que han tomado parte en el artículo. De todas maneras, esto no es más que una hipótesis por comprobar.

Por otro lado, para el estudio de la cooperación entre centros de investigación se tuvo que recurrir, al contrario que en la prueba anterior, a los resultados obtenidos en las bases de datos SCI e IME. La razón estriba en la escasez de información de algunos campos de Medline; por ejemplo, en el caso de la dirección, sólo incluye la del primer autor.

El estudio realizado pretende establecer el grado de cooperación entre centros de investigación tanto a nivel nacional como internacional. El bajo número de colaboraciones, si se compara con el índice de coautoría analizado anteriormente, hace pensar que, en realidad, el alto número de autores se debe al volumen de los equipos de investigación dentro de una misma institución.

De igual modo, otro aspecto a destacar es la proximidad geográfica de la cooperación entre los centros de investigación. Existen muchos más documentos producidos en colaboración entre centros españoles que entre centros españoles y extranjeros (véase tabla VIII); aunque los más numerosos son los artículos escritos en colaboración entre científicos del mismo centro, pero de unidades distintas.

La colaboración con centros de otros países se da, sobre todo, en los organismos de investigación de dos ciudades: Barcelona y Madrid. De la misma forma, se debe resaltar que aquellos documentos en los que existe colaboración extranjera cuentan con un número mayor de centros participantes.

Si se observa la red de colaboración entre distintas ciudades, se verá cómo la colaboración entre ciudades también es mínima. Incluso así, el binomio Barcelona - Madrid se hace patente como eje principal de la red de cooperación de este país. Además 
Tabla VIII

Nivel-de cooperación entre centros de investigación Datos de SCI e IME

\begin{tabular}{|c|c|c|c|}
\hline Año & $\begin{array}{c}\text { Números de documentos } \\
\text { producidos en colaboración }\end{array}$ & $\begin{array}{c}\text { Colaboración centre centros } \\
\text { españoles y extranjeros }\end{array}$ & $\begin{array}{c}\text { Colaboración entre centros } \\
\text { españoles }\end{array}$ \\
\hline 1990 & 42 & $17,59 \%$ & $82,41 \%$ \\
1991 & 50 & $27,4 \%$ & $72,6 \%$ \\
1992 & 81 & $14,17 \%$ & $85,83 \%$ \\
1993 & 93 & $8,03 \%$ & $91,97 \%$ \\
1994 & 82 & $16,42 \%$ & $83,58 \%$ \\
1995 & 119 & $9,44 \%$ & $90,56 \%$ \\
1996 & 127 & $11,01 \%$ & $88,99 \%$ \\
\hline
\end{tabular}

de este eje, se encuentran dos triángulos de producción: Barcelona - Madrid - Valencia y Barcelona - Madrid - Santander. En estos casos, se observa cómo los criterios de proximidad geográfica están presentes: Barcelona tiene mayor colaboración con Valencia que Madrid; y Madrid una mayor cooperación con Santander que esta ciudad con Barcelona. Los dos triángulos restantes, Barcelona - Madrid - Córdoba y Madrid - Córdoba - Cádiz, son de menor producción. Por último, y como curiosidad, hay que resaltar la presencia de la ciudad de Oviedo, que se une a la red en el eje cordobés. Resulta sorprendente que esta ciudad no se una al eje a través de una colaboración con ciudades de tradición productora en este tema y que están más próximas geográficamente: por ejemplo, Santander o Madrid.

Por último, sorprende la ausencia de ciudades con una producción importante como Pamplona, Murcia y A Coruña; que aunque se comportan como núcleos de investigación no entran en la red principal de cooperación investigadora. Por contra, ciudades como Cádiz u Oviedo, se aprovechan de esta red para mantener un cierto ritmo de publicación.

\subsection{Idiomas y países donde se publica}

El análisis de los idiomas de publicación puede parecer poco revelador cuando se analiza, entre otras, una base de datos de acusada tradición anglosajona (SCI) y un área temática que ha recogido, desde hace tiempo, el inglés como lengua común de intercambio científico (16). Sin embargo, si se estudian estos idiomas junto a los países en los que los artículos han sido publicados, se pueden apreciar algunos elementos de juicio interesantes.

Existe una división clara entre los artículos publicados en inglés y los publicados en español, mostrando una dicotomía clara entre revistas internacionales y nacionales. En el caso nacional, la correspondencia entre lugar de publicación e idioma es casi matemática: podría decirse que, en este país, no se publica casi nada en una lengua que no sea la propia. Algunas de nuestras publicaciones incluyen abstracts y descriptores en lengua inglesa, pero no han dado el paso de publicar directamente en inglés; aspecto que, a pesar del perjuicio que recibiría el idioma español, seguramente subiría el factor de impacto de esas publicaciones. 


\subsection{Distribución por revistas de los trabajos}

Dentro del período estudiado, IME y Medline indizaron 1.814 artículos distintos publicados en 326 revistas diferentes. El corazon de este conjunto documental (véase tabla IX) está formado por 10 publicaciones $(3,06 \%)$ que recogen 920 artículos $(50,71 \%)$. Los datos recogidos marcan una dispersión muy alta ( $\mathrm{y}$ de valor superior a la expresada por M. A. Pérez San-Gregorio et al. (9), que exponían que en un $8,58 \%$ de las publicaciones se encontraba el $43,26 \%$ de los documentos), factor que, según Lawani (17), perjudicaría la calidad de los trabajos. Esta diseminación en la publicación sigue la tónica de la producción española en el área de genética (13) y en el área de inmunología (18); lo que nos puede dar la idea de una característica endémica de la productividad nacional.

Tạbla IX

Conjunto de revistas donde más documentos españoles sobre transplantes han sido publicados Datos de MEDLINE e IME

\begin{tabular}{|l|c|}
\hline \multicolumn{1}{|c|}{ Revista } & $\begin{array}{c}\text { Núm. de articulos } \\
\text { publicados }\end{array}$ \\
\hline Transplantation Proceedings & 272 \\
Revista Española de Trasplantes & 128 \\
Nefrologia & 101 \\
Revista Española de Cardiología & 73 \\
Bone Marrow Transplantation & 67 \\
Medicina Clínica & 66 \\
Cirugia Española & 64 \\
Archivos Españoles de Urologia & 59 \\
Actas Urológicas Españolas & 50 \\
Transplantation & 40 \\
\hline
\end{tabular}

\subsection{Análisis de las referencias}

Se ha realizado un análisis de la información disponible en el JCR y la base de datos SCI para conocer la actualización de la bibliografía utilizada por los autores españoles, poniéndola en relación con sus colegas extranjeros. Los indicadores seleccionados han sido la vida media y el índice de Price.

Por otro lado, con la intención de comparar las tendencias de publicación de las revistas nacionales con las internacionales, se ha realizado un estudio sobre la ventana de citación de dichos nichos de publicación.

En la tabla $\mathrm{X}$ se muestran los datos utilizados para comparar la producción española con la producción internacional. Para ello se han tenido en cuenta diversos factores: en primer lugar, se han recogido los datos de una revista paradigmática (la revista con mayor factor de impacto pero desechando las dedicadas a revisiones, por el sesgo que aportan), y, en segundo lugar, se han separado las revistas donde publican los científicos españoles según el ámbito de publicación. De este modo se intenta descubrir si es cierta la idea de que, cuando se publica en revistas internacionales, las tendencias de publicación son diferentes a cuando los artículos aparecen en una publicación de nivel nacional. 
Tabla X

Vida media e índice de Price de la bibliografía consultada por los científicos españoles

Datos de SCI y JCR

\begin{tabular}{|l|c|c|c|}
\hline \multicolumn{1}{|c|}{ Revista o ámbito de publicación } & $\begin{array}{c}\text { Artículos } \\
\text { publicados }\end{array}$ & $\begin{array}{c}\text { Vida } \\
\text { media }\end{array}$ & $\begin{array}{c}\text { Indice de } \\
\text { Price }\end{array}$ \\
\hline Revista paradigmática de la disciplina (Transplantation) & 40 & 4,64 & $62,04 \%$ \\
Artículos publicados en el ámbito nacional & 911 & 5,66 & $52,99 \%$ \\
Artículos publicados en el ámbito internacional & 903 & 5,58 & $53,86 \%$ \\
\hline
\end{tabular}

La comparación de la producción española con la revista paradigmática refleja una obsolescencia más pronunciada, tanto en el caso de la vida media como en el índice de Price.

Así, según la teoría de Price (19), los documentos publicados por autores españoles no forman un frente de investigación; ya que los valores de vida media e índice de Price de estos documentos están muy distantes de los de la revista paradigmática de la disciplina.

De todos modos, se observa que los científicos españoles eligen, como revistas donde enviar sus trabajos, publicaciones con una vida dilatada y notable influencia, sean del ámbito que sean (por ejemplo: Transplantation Proceedings o Revista Española de Cardiología).

Por otro lado, se ve también que la dispersión en la publicación perjudica los valores de los indicadores estudiados: si se publicase más en estas revistas que ya son de por sí las más utilizadas, los valores medios serían todavía mejores y tendrían una menor diferencia respecto a la revista paradigmática.

Otro aspecto a comentar es la no diferencia en las tendencias de citación, en lo que a actualización de las fuentes se refiere, en función del ámbito de publicación. Cuando se publica en el extranjero, los valores medios son algo mejores, aunque la diferencia no es realmente significativa. Incluso, si se observa la figura 4 , se aprecia cómo los valores de la ventana de citación son muy parecidos; pero si se estudia la bibliografía más actualizada (de uno o dos años de antigüedad), se ve que es superior en los artículos publicados en España.

\section{Conclusiones}

Por todo lo dicho en el estudio, parece que la producción española en el campo de los transplantes está por debajo de lo que cabría esperar (a pesar de la importancia que se le otorga), aunque se da una tendencia al alza. Como ejemplos se apuntan la producción total, que sube rápidamente, sobre todo en el aspecto internacional, y el indicador de ganancia en competitividad, que muestra unos valores muy positivos, tanto en la comparación con el resto de países como en la propia evolución española.

Este aumento de producción y competitividad podría entenderse como un aumento en la calidad. Y aunque las teorías de Price desmienten este hecho, la elección de publicaciones realizada por los autores españoles es realmente buena: todas las revistas extranjeras donde aparecen estos artículos poseen valores muy significativos dentro del SCI. A pesar de estos aspectos sería necesario reducir la excesiva dispersión temática 
Figura 4

Ventana de antigïedad de la bibliografía utilizada

Datos de SCI

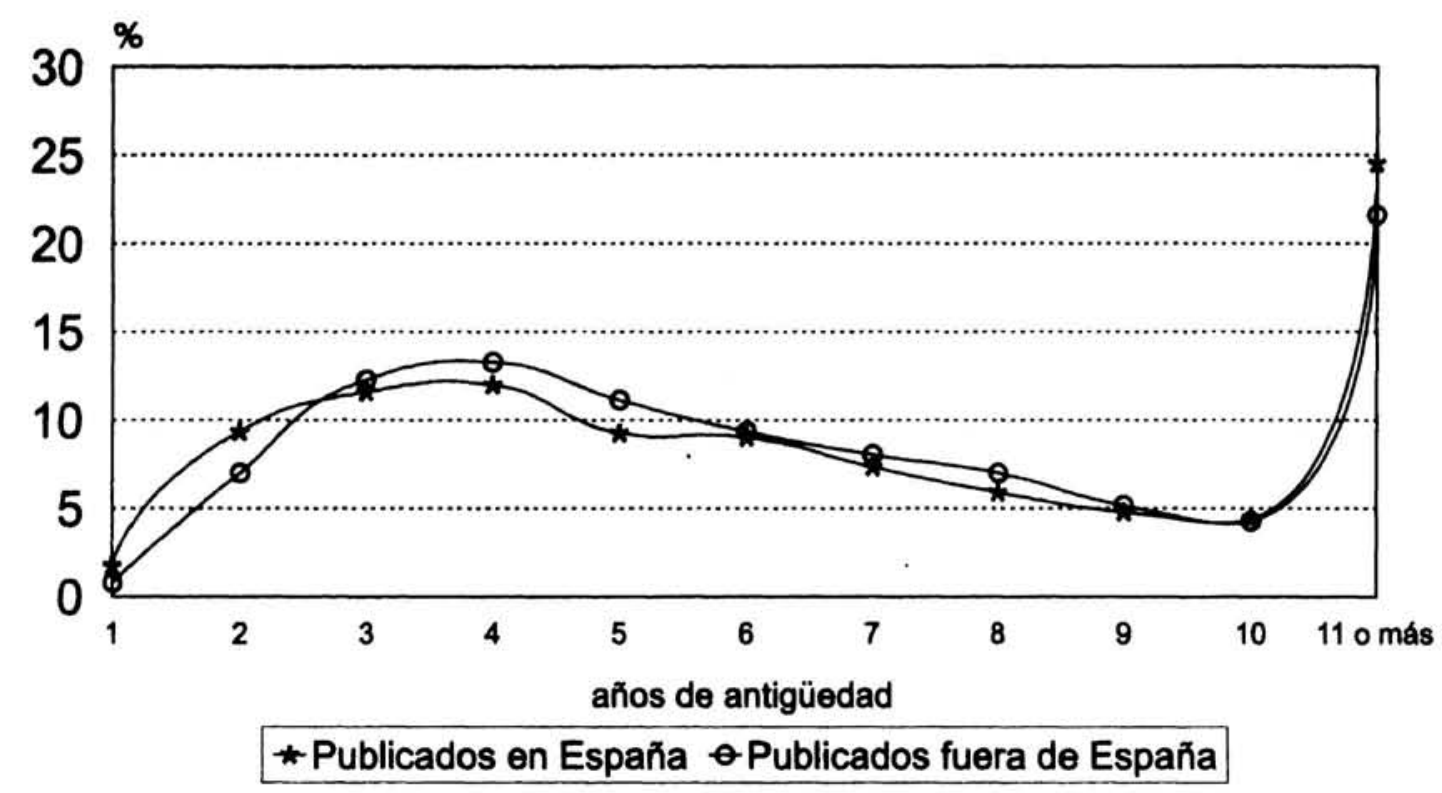

y de publicación existente: una mayor concentración mejoraría los resultados (17) y (20). Asimismo, el hecho de que en España sólo se publique en español también baja el factor de impacto de las revistas nacionales.

Además, se observa la necesidad de estudiar el destino concreto de los fondos invertidos en investigación médica; ya que, aunque parece existir una correlación entre recursos económicos y producción científica, esta correlación no se da en todas las comunidades autónomas del país. Esto puede deberse a que la temática presenta diferencias de prioridad de unas comunidades a otras. Así, sería necesario estabilizar la financiación como solución a la inmadurez investigadora y el desequilibrio del reparto, dejando de lado otros intereses (11) y (21).

Por otro lado, se colabora bastante menos de lo que cabría esperar. Los centros no parecen colaborar entre sí; y tan sólo las instituciones de Barcelona y Madrid colaboran con el extranjero. De igual modo, es remarcable la diferencia de los niveles de coautoría según donde se publica.

Asimismo, no se distinguen diferencias en el uso de información bibliográfica por parte de los investigadores teniendo en cuenta el ámbito de publicación de su estudio. Es decir, las costumbres de mención de bibliografía, en cuanto a la actualización de las fuentes, son prácticamente idénticas; y no se da el hecho de que se cite bibliografía más actualizada cuando se publica en una revista internacional que cuando se publica en una de ámbito nacional.

\section{Bibliografía}

1. MIRANDA, B. Más vidas que renacen. Anuario de la salud, la medicina y la sanidad 1998. Madrid: Fomento Salud, cop. 1998. 
2. MATESANZ, R. El transplante y los medios de comunicación. Revista Española de Trasplantes, 1993, 2 (4), pp. 179-183.

3. MINISTERIO DE SANIDAD Y CONSUMO. Fondo de Investigación Sanitaria. Fondo de Investigación Sanitaria: memoria [1988-1995]. Madrid: Instituto Nacional de la Salud, 1989-1996.

4. CANO, F.; JULIÁN, S. Some indicators in Spanish scientific production. Scientometrics, 1992, 24, pp. 43-59.

5. FRAME, J. D. Mainstream research in Latin America and the Caribbean. Interciencia, 1977, 2, pp. 143-148.

6. GUZMÁN, M. V.; SANZ, E.; SOTOLONGO, G. Bibliometric study on vaccines (19901995), part I: Scientific production in Iberian-American countries. Scientometrics, 1998, 43, pp. 189-205.

7. SCHUBERT, A.; BRAUN, T. Relative indicators and relational chart for comparative assessment of publication output and citation impact. Scientometrics, 1986, 14, pp. 281-291.

8. NAGPAUL, P. S.; SHARMA, L. Science in the eighties: a typology of countries based in inter-field priorities. Scientometrics, 1995, 34, pp. 263-283.

9. PÉREZ SAN-GREGORIO, M. A., et al. Investigaciones psicológicas sobre las donaciones y trasplantes: un estudio bibliométrico. Cuadernos de medicina psicosomática, 1994, 32 , pp. 14-25.

10. GOLDBERG, A. I.; OIGENBLICK, L.; RUBIN, A. H. E. Scientific articles and national medical cultures: a comparison of Russian and American medical journals. Scientometrics, 1997, 39, pp. 57-75.

11. BAREA TEJEIRO, J. Escenarios financieros de la sanidad española. Anuario de la Salud, la Medicina y la Sanidad 1998. Madrid: Fomento Salud, cop. 1998.

12. CORTÉS GUARDIOLA, J. A. Un decenio de trasplantes: análisis de la producción científica española sobre trasplantes de riñón, hígado, corazón, pulmón y cardiopulmonar a través del IME durante el periodo 1982-1991. Tesis doctoral, Universidad de Murcia, 1994.

13. MARTÍN MORENO, C.; SANZ, E. Producción científica española en el área de genética. Revista española de documentación cientifica, 1996, 19, pp. 377-391.

14. LAWANI, S. M., et al. Quality, collaboration and citations in cancer research: a bibliometric study. Michigan: Ann Arbor, 1980.

15. PÉREZ SAN GREGORIO, M.A., et al. Estudio de revisión de la literatura (1974-1988) sobre los aspectos psicológicos implicados en las donaciones y trasplantes de órganos. $\mathrm{Ne}$ frología, 1991, 11 (supl. 1), pp. 69-75.

16. VILLAR, J. El inglés, idioma internacional en medicina. Medicina Clínica, 1988, 91, pp. 23-24.

17. LAWANI, S. M. Some bibliometric correlates of quality in scientific research. Scientometrics, 1986, 9, pp. 13-25.

18. ARAGÓN GONZÁLEZ, I. Análisis bibliométrico de la producción científica española en inmunologia: periodo 1980-1992. Tesis doctoral, Universidad Complutense de Madrid, 1995. Citado por C. Martín Moreno y E. Sanz, Producción científica española en el área de genética, Revista española de documentación científica, 1996, 19, pp. 377-391.

19. PRICE, D. J. S. Networks of scientific papers. Science, 1965, 149, pp. 510-515.

20. TERRADA, M. L. La actividad científica en la España actual: análisis de las publicaciones. VIII Reunión Científica del Fondo de Investigación Sanitaria. Madrid: Instituto Nacional de la Salud, 1991.

21. LÓPEZ CASANOVAS, G. El puzzle de la política sanitaria en España. Anuario de la Salud, la Medicina y la Sanidad 1998. Madrid: Fomento Salud, cop. 1998. 